\title{
Praxis der Sterbehilfe durch Ärzte und Pflegekräfte in deutschen Krankenhäusern
}

\section{Practice of euthanasia among physicians and nurses in German hospitals}

Autor

Karl H. Beine

\section{(ㄷ) (1) $(9)$}

Institut

Lehrstuhl für Psychiatrie und Psychotherapie,

Universität Witten/Herdecke (bis 2019)

\section{Schlüsselwörter}

Sterbehilfe, assistierter Suizid, Tötung ohne explizite

Willensäußerung

Key words

euthanasia, assisted suicide, killing without explicit request

online publiziert 13.10 .2020

Bibliografie

Dtsch Med Wochenschr 2020; 145: e123-e129

DOI 10.1055/a-1235-6550

ISSN $0012-0472$

(C) 2020. The Author(s).

This is an open access article published by Thieme under the terms of the Creative Commons Attribution-NonDerivative-NonCommercial License, permitting copying and reproduction so long as the original work is given appropriate credit. Contents may not be used for commecial purposes, or adapted, remixed, transformed or built upon. (https://creativecommons.org/licenses/by-nc-nd/4.0/)

Korrespondenzadresse

Prof. Dr. med. Karl H. Beine

St.-Marien-Hospital Hamm, Nassauer Straße 13-19, 59065 Hamm, Deutschland

Karl.Beine@uni-wh.de

Zusätzliches Material finden Sie unter https://doi.org/10.1055/a-1235-6550

\section{ZUSAMMENFASSUNG}

Hintergrund Aktive Sterbehilfe und ärztlich assistierter Suizid werden in Deutschland kontrovers diskutiert. Empirische Studien fehlen, um ihr jeweiliges Vorkommen, einschließlich dem von passiver und indirekter Sterbehilfe, in deutschen Krankenhäusern einschätzen zu können. Ärztinnen und Ärzte sowie Pflegerinnen und Pfleger in deutschen Krankhäusern wurden zu ihrer Anwendung von Sterbehilfe befragt und mögliche Einflussfaktoren erhoben.

Methode Angaben zu Sterbehilfe wurden mit deskriptiven Begriffen und zugeordneten Definitionen in einer anonymen
Online-Befragung erhoben. Die objektive und subjektive Arbeitssituation der Befragten und ihre Befürwortung von aktiver Sterbehilfe wurden erfasst. Die finale ärztliche Stichprobe umfasste $n=2507$, die pflegerische Stichprobe umfasste $\mathrm{n}=2683$ Personen.

Ergebnis Die Anwendung von passiver und indirekter Sterbehilfe innerhalb von 24 Monaten wurde von einem großen Anteil der ärztlichen und pflegerischen Befragten angegeben, aktive Sterbehilfe und assistierter Suizid von deutlich weniger Befragten. Die Varianz in der Anwendung von aktiver Sterbehilfe wurde $u$. a. durch mehrere arbeitsbezogene Faktoren und die jeweilige Befürwortung von aktiver Sterbehilfe beeinflusst, nicht aber durch subjektive Belastungsfaktoren.

Schlussfolgerung Sterbehilfe wird durch ärztliche und pflegerische Mitarbeiterinnen und Mitarbeiter in deutschen Krankenhäusern praktiziert. Das Vorkommen unterschiedlicher Formen von Sterbehilfe und relevante Einflussfaktoren werden vor dem Hintergrund methodischer Limitationen diskutiert.

\section{ABSTRACT}

Background Active euthanasia and physician-assisted suicide are controversially discussed in Germany. Empirical studies are missing to estimate their respective incidence, including passive and indirect euthanasia in German hospitals. Physicians and nurses in German hospitals were surveyed regarding their practice of euthanasia and possible influence factors.

Method Information on euthanasia practice was obtained using descriptive terms and related definitions in an anonymous online survey. Participants' objective and subjective occupational situations and support of euthanasia were recorded. The final samples comprised $\mathrm{N}=2507$ physicians and $\mathrm{N}=2683$ nurses.

Result The practice of passive and indirect euthanasia was reported by a large number of physicians and nurses during the last 24 months, active euthanasia and assisted suicide was reported by substantially less participants. Variance among the practice of active euthanasia could be explained by occupational factors and the respective advocacy of euthanasia amongst other variables, but not by subjective burden.

Discussion Euthanasia is practiced by physicians and nurses in German hospitals. The Incidence of different types of euthanasia and relevant influence factors are discussed considering methodical limitations. 


\section{Hintergrund}

Die öffentliche Debatte um verschiedene Formen der sogenannten Sterbehilfe ${ }^{1}$ in Deutschland wird seit Jahren intensiv geführt. Gerichtsprozesse zum Thema befeuern regelmäßig gesellschaftliche Kontroversen, zuletzt anlässlich der Entscheidung des Bundesverfassungsgerichts vom Februar 2020. Demnach umfasst das allgemeine Persönlichkeitsrecht ein Recht auf selbstbestimmtes Sterben und die Freiheit, hierfür bei Dritten Hilfe zu suchen und in Anspruch zu nehmen [1]. Auch die davon abzugrenzende aktive Sterbehilfe, welche die intentionale Tötung eines z. B. schwerkranken Patienten auf dessen expliziter freien Willensbekundung bezeichnet (siehe Kasten 1), hat Befürworter. Diese verweisen auf die in den Beneluxstaaten gesetzlichen Verfahren zur straffreien Ausübung von aktiver Sterbehilfe [2]. Die Mehrheit der Ärzteschaft in Deutschland steht dem jedoch ablehnend gegenüber und verweist auf die bestehenden Möglichkeiten der Palliativmedizin, welche Todeswünsche bei Patientinnen und Patienten reduzieren können [3] und unbeabsichtigt als passive und indirekte Sterbehilfe zum Tod führen können.

In welchem Umfang und durch wen verschiedene Formen von Sterbehilfe in Deutschland tatsächlich durchgeführt werden, ist bisher nur unzureichend erforscht. Die wenigen Studien zur Sterbehilfe in Deutschland liefern heterogene Befunde: Ergab die Befragung niedergelassener Hausärztinnen und -ärzte, jemals „aktive Sterbehilfe“ durchgeführt zu haben, eine Zustimmung von $13 \%$ [4], waren es bei einer anderen Frageformulierung und -methode innerhalb von 12 Monaten nur 0,27\% der palliativmedizinisch tätigen Ärztinnen und Ärzte [5]. Die Unterschiede in Itemformulierung, Erfassungszeitraum, Stichprobe und Fragebogenkonstruktion erschweren Vergleichbarkeit und Verlässlichkeit dieser Befunde. Dabei wird insbesondere bei Studienergebnissen zur Sterbehilfe auf die starke Abhängigkeit der angewandten Methodik hingewiesen [6]. Zum einen kann die Verwendung bekannter, jedoch uneindeutiger Begriffe wie „Sterbehilfe“ [4] oder eine unspezifische Wortwahl [7] eine falsche Zuordnung zu den verschiedenen Formen von Sterbehilfe bewirken, die sogar unter Fachkräften überraschend hoch ist [8]. Zum anderen kann bereits die Reihenfolge der gestellten Fragen [9] und inhaltliche Überschneidung der erfragten Kategorien zu Verzerrungen führen [5, 10].

Noch weniger ist über Einflussfaktoren bekannt, die die Durchführung aktiver Sterbehilfe in Deutschland begünstigen oder verringern. Die bisherigen, teils inkonsistenten Befunde aus anderen Ländern legen nahe, dass Behandelnde wahrscheinlich verstärkt aktive Sterbehilfe durchführen, wenn sie männlich und höheren Alters sind, eine palliativmedizinische Ausbildung sowie Erfahrung mit todkranken Patienten haben [11, 12] und nicht religiös-fundierte Standpunkte vertreten bzw. die aktive Sterbehilfe direkt befürworten [11, 13]. Andere Studien deuten hingegen darauf hin, dass eine palliativmedizinische Weiterbildung Behandelnden alternative Handlungsoptionen vermittelt, um auf die Durchfüh-

1 Der Begriff der Sterbehilfe wird vielfach als unspezifisch kritisiert. Da sich bislang jedoch keine allgemein akzeptierten Alternativen durchgesetzt haben, wird er in dieser Studie als Oberbegriff verwendet und spezifiziert (siehe Kasten 1). rung von aktiver Sterbehilfe zu verzichten [14]. Keine empirischen Erkenntnisse liegen bislang zu der von Patientinnen und Patienten geäußerten Befürchtung vor, die mit Unterbesetzung und Arbeitsbelastung einhergehende erhöhte Patientenmortalität [15] könne sich angesichts von Pflegenotstand und Ärztemangel in deutschen Krankenhäusern auch auf die Praxis von aktiver Sterbehilfe auswirken. Zudem wurden in keiner der bisherigen Studien in Deutschland relevante Einflussfaktoren gezielt mit dem dafür nötigen Stichprobenumfang untersucht.

\section{DEFINITIONEN DER SOG. STERBEHILFE}

„Passive Sterbehilfe“ bezeichnet das Zurückhalten/den Entzug einer lebenserhaltenden oder -verlängernden Behandlung (z. B. künstliche Beatmung, Ernährung oder (weitere) Gabe eines Medikaments) nach entsprechender Einwilligung des/der Patienten/-in, dessen/deren erfolgter Tod nicht gewollt, sondern eine unbeabsichtigte oder in Kauf genommene Folge darstellt.

"Indirekte Sterbehilfe“ bezeichnet die Gabe eines Medikaments (z. B. Opioide, Benzodiazepine, Barbiturate) zur Schmerzlinderung nach entsprechender Einwilligung des/der Patienten/-in, dessen/deren erfolgter Tod nicht gewollt, sondern eine unbeabsichtigte oder in Kauf genommene Folge darstellt.

„Assistierter Suizid“, häufig wird „ärztlich“ vorangestellt, bezeichnet die Aushändigung eines Medikaments an eine/n Patienten/-in zur selbstständigen Beendigung seines/ihres Lebens. "Aktive Sterbehilfe“ bezeichnet aktive Handlungen (Behandlungen, Interventionen etc.), die eine aktive Beendigung des Lebens eines/-r Patienten/-in beabsichtigen bzw. zum Ziel haben.

Zudem wird hier unterschieden zwischen:

- „Tötung auf Verlangen“ (wurde in der Befragung nicht begrifflich eingeführt, jedoch anhand der Angaben zum letzten Fall von aktiver Sterbehilfe erhoben) nach diesbezüglicher expliziter Willensäußerung des/der Patienten/-in wird häufig juristisch synonym verwendet.

" „Tötung ohne explizite Willensäußerung“ wird üblicherweise nicht der aktiven Sterbehilfe zugeordnet, ist in einigen Fällen jedoch schwer abgrenzbar.

Zur Vermeidung von falschen Zuordnungen wurden bei jeder Nennung der jeweiligen deskriptiven Begriffe (kursiv) während der Umfrage die oben genannten Definitionen (grammatikalisch angepasst) eingeblendet.

Ziel der vorliegenden Studie war es, in deutschen Krankenhäusern tätige Ärztinnen und Ärzte gemeinsam mit Pflegerinnen und Pflegern zu allen von ihnen praktizierten Formen von Sterbehilfe und möglichen Einflussfaktoren auf aktive Sterbehilfe zu befragen. Um verlässliche Daten zu erhalten, wurde die Erhebung unter für Befragte transparenter Anonymisierung und neutraler Fragebogenformulierung durchgeführt. 


\section{Methode}

\section{Fragebogen und Variablen}

Der Fragebogen (s. Online-Anhang) bestand aus mehreren neu konstruierten Skalen, welche auf Basis einer kritischen Literaturschau und von Erhebungsinstrumenten aus vorangegangenen Studien zur Sterbehilfe [16] an die Arbeitssituation in Krankenhäusern angepasst wurden. Nach einem Pretest wurde der Fragebogen geringfügig modifiziert. Der einleitende Text zum Inhalt der Befragung betonte die Anonymität und Freiwilligkeit aller Angaben sowie den jederzeit möglichen Abbruch. Die Fragen bezogen sich mehrheitlich auf die vergangenen 24 Monate.

\section{Formen von Sterbehilfe}

Die verschiedenen Formen von Sterbehilfe wurden separat definiert, mit Beispielen versehen und in der Befragung beschrieben. Zur Vermeidung von falschen Zuordnungen wurde bei jeder betreffenden Frage die zugehörige Definition der jeweiligen Form von Sterbehilfe eingeblendet (in Kasten 1 kursiv). Zu Beginn wurden die für Sterbehilfe relevanten (Arbeits-) Bedingungen erfragt, u. a. die Frequenz des Kontakts zu sterbenden Patientinnen und Patienten und ihres geäußerten Wunsches nach aktiver Sterbehilfe. Die Fragen zur eigenen Praxis von Sterbehilfe wurden nach absteigendem Risiko potenzieller Antwortverzerrung angeordnet: Als erstes wurde die bei anderen, z. B. Kolleginnen und Kollegen beobachtete und die selbst durchgeführte aktive Sterbehilfe erfragt, für die vergangenen 24 Monate und die gesamte bisherige Tätigkeit: „Wie häufig haben Sie selbst das Leben von Patienten/-innen aktiv beendet?" 2 Die Befragten, die aktive Lebensbeendigung jemals praktiziert hatten, wurden jeweils gebeten, die Anzahl der Fälle zu benennen und anzugeben, durch welche Maßnahmen die aktive Lebensbeendigung im letzten Fall erreicht wurde.

Anschließend wurde nach der eigenen Durchführung und entsprechender Fallzahl von anderen Formen von Sterbehilfe (passiv, indirekt, assistierter Suizid, andere Methoden) gefragt. Abschließend wurde auf einer 5-stufigen Skala von 1 („stimme überhaupt nicht zu“) bis 5 („stimme voll und ganz zu“) erfragt, inwieweit eine Straffreiheit für Ärztinnen und Ärzte bzw. Pflegerinnen und Pfleger bei Durchführung aktiver Sterbehilfe (mit oder ohne explizite Willensäußerung) befürwortet wird (gute interne Konsistenz der Skala, $\alpha=80$ ).

\section{Subjektive Belastungsfaktoren}

Subjektive Belastungsfaktoren wurden durch Fragen zur Arbeitsbelastung (z. B. „Bei der Menge an Aufgaben bleibt für die Patientenversorgung meist zu wenig Zeit übrig.“), Unterstützung im Team (z. B. „Von meinem Team bekomme ich Unterstützung und Hilfe, wenn ich sie brauche.") und zur beruflichen Zufriedenheit insgesamt (u.a. mit Anforderungen, Betriebsklima, Bezahlung) erhoben. Jede der 3 Subskalen bestand aus 7-8 Items mit einer

2 "Aktive Sterbehilfe« war im Fragebogen wie folgt definiert: Aktive Handlungen (Behandlungen, Interventionen, etc.), die eine Beendigung des Lebens beabsichtigen bzw. zum Ziel haben. Zur Vermeidung von begrifflichen Unklarheiten werden im Folgenden alle entsprechenden Handlungen als Leben aktiv beenden bezeichnet. 5-stufigen Skala von 1 („trifft überhaupt nicht zu“) bis 5 („trifft voll und ganz zu“ bzw. von „unzufrieden“ bis „zufrieden“). Alle Skalen wiesen zufriedenstellende Gütekriterien auf, darunter interne Konsistenzen von $\alpha=0,80-0,87$.

\section{Soziodemografische Angaben und Arbeitssituation}

Soziodemografische und arbeitsbezogene Angaben konnten zu Beginn der Befragung freiwillig und unter Wahrung der Anonymität angegeben werden. Zu den Angaben zählten u. a. Berufsgruppe, Geschlecht, Alter, Berufserfahrung, Familienstand und Fachgebiet sowie das Tätigkeitsfeld Krankenhaus, um die Stichprobe darauf beschränken zu können. Zum Abschluss der Befragung waren Kommentare und die Bitte um Löschung der Daten möglich.

\section{Erhebung und Stichproben}

Ausschließlich in deutschen Kliniken beschäftigte Ärzte und Ärztinnen sowie Pfleger und Pflegerinnen nahmen im Zeitraum von September bis Dezember 2018 an einer identischen anonymen Online-Befragung teil. Ärztinnen und Ärzte wurden bundesweit über die Kooperation mit einem Adressenverlag per E-Mail angeschrieben und 2-mal erinnert. Pflegerinnen und Pfleger wurden über eine berufsspezifische Anzeige in einem sozialen Netzwerk, über einen Hinweis im Newsletter des Deutschen Berufsverbandes für Pflegeberufe und im Pflegeportal Bibliomed um eine Teilnahme an der Befragung gebeten. 5386 Personen beendeten den Fragebogen. Befragte wurden ausgeschlossen, wenn sie einer anderen als der ärztlichen oder pflegerischen Berufsgruppe angehörten ( $n=46)$ oder einen anderen Arbeitsplatz als das Krankenhaus ( $n=144)$ angaben sowie bei Verständnisproblemen, Bitte um Löschung ( $n=2)$ und im Zuge der Datenbereinigung $(n=4)$, sodass final die ärztliche Stichprobe $n=2507$ und die pflegerische Stichprobe $\mathrm{n}=2683$ umfasste.

Die Verteilung beider Stichproben auf die unterschiedlichen medizinischen Fachgebiete sind in $>$ Tab. 1 dargestellt, soziodemografische Angaben in > Tab. 2. Das höhere ärztliche Durchschnittsalter und entsprechende Berufserfahrung verdeutlichen vorhandene soziodemografische Unterschiede zwischen den Stichproben. Die Verteilung beider Stichproben auf die jeweiligen Fachgebiete war dagegen ähnlicher ( $\vee$ Tab. 1).

Die Studie wurde von der Ethik-Kommission der Universität Witten/Herdecke genehmigt (Referenznr.: 93/2018).

\section{Ergebnisse}

Statistische Analysen, Datenbereinigung und Ermittlung fehlender Werte $(0,24 \%)$ wurden mit IBM SPSS Statistics 26 durchgeführt. Die Voraussetzungen der statistischen Verfahren wurden jeweils überprüft und waren gegeben: Die Normalverteilung der verwendeten Variablen war entweder gegeben oder verwendete Tests waren aufgrund des hohen Stichprobenumfangs deren Verletzung gegenüber robust. Vor der durchgeführten Regressionsanalyse konnte Multikollinearität ausgeschlossen werden und Linearität gezeigt werden.

Alle Angaben bezogen sich, wenn nicht anders beschrieben, auf die vergangenen 24 Monate. 
- Tab. 1 Fachgebiet bzw. Arbeitsschwerpunkt der letzten 24 Monate in beiden Stichproben.

\begin{tabular}{|c|c|c|}
\hline \multirow[t]{2}{*}{ Fachgebiet } & $\begin{array}{l}\text { Ärztinnen } \\
\text { und Ärzte }\end{array}$ & $\begin{array}{l}\text { Pflegerinnen } \\
\text { und Pfleger }\end{array}$ \\
\hline & $n(\%)$ & $n(\%)$ \\
\hline Innere Medizin & $408(16,29)$ & $803(29,97)$ \\
\hline Chirurgie & $655(26,16)$ & $619(23,11)$ \\
\hline Allgemeinmedizin & $5(0,20)$ & $82(3,06)$ \\
\hline Anästhesie/Intensiv/Notfall & $329(13,14)$ & $323(12,06)$ \\
\hline Neurologie/Psychiatrie & $372(14,86)$ & $329(14,63)$ \\
\hline Gynäkologie & $176(7,03)$ & $58(2,16)$ \\
\hline Urologie & $70(2,80)$ & $45(1,68)$ \\
\hline HNO & $39(1,56)$ & $19(0,71)$ \\
\hline Dermatologie & $39(1,56)$ & $11(0,41)$ \\
\hline Radiologie/Nuklearmedizin & $99(3,95)$ & $9(0,34)$ \\
\hline Palliativmedizin & $48(1,92)$ & $57(2,13)$ \\
\hline Pädiatrie (inkl. Onkologie) & $207(8,27)$ & $149(5,56)$ \\
\hline $\begin{array}{l}\text { Interdisz./Ausbildung/ } \\
\text { wechselnd }\end{array}$ & $0(0,00)$ & $89(3,32)$ \\
\hline Sonstige & $57(2,28)$ & $23(0,86)$ \\
\hline Gesamt, n & $2507(100,00)$ & $2683(100,00)$ \\
\hline $\begin{array}{l}\text { Stichprobenumfang überste } \\
\text { gebiete aufgrund von fehlen } \\
\text { lende Werte unberücksichti }\end{array}$ & $\begin{array}{l}\text { e Summe der } \\
\text { Angaben. Bei P }\end{array}$ & $\begin{array}{l}\text { gkeiten der Fach- } \\
\text { twerten sind feh- }\end{array}$ \\
\hline
\end{tabular}

\section{Objektive und subjektive Arbeitssituation}

Der regelmäßige Kontakt zu sterbenden Patientinnen und Patienten lag bei Pflegerinnen und Pflegern ([P] mind. 1-mal wöchentlich $=39,82 \%$ ) deutlich höher als bei Ärztinnen und Ärzten $([\ddot{A}]=4,70 \%)$. Pflegerinnen und Pfleger wurden auch häufiger, meist durch die Patientinnen und Patienten selbst, um aktive Sterbehilfe gebeten (mind. 1-mal in 24 Monaten [P] = 50,38\%; $[\ddot{A}]=29,21 \%)$. Ärztinnen und Ärzte gaben im Berufsgruppenvergleich eine geringere subjektive Belastung auf allen erfragten Dimensionen an (alle Unterschiede signifikant, siehe $>$ Tab. 2).

\section{Praxis von Sterbehilfe bei ungewolltem Tod}

Über die Hälfte der Ärztinnen und Ärzte sowie mehr als ein Drittel der Pflegerinnen und Pfleger berichteten für die vergangenen 24 Monate von Interventionen, die ungewollt zum Tode des/der Patienten/-in führten oder diesen in Kauf nahmen ( $\vee$ Tab.3). Gemessen an allen Fällen von Sterbehilfe in den vergangenen 24 Monaten machten passive und indirekte Sterbehilfe in beiden Berufsgruppen einen Anteil von mehr als $90 \%$ aus ( $\triangleright$ Tab. 4).

Assistierter Suizid wurde im Vergleich seltener, jedoch häufiger von Pflegerinnen und Pflegern berichtet. 15 Befragte gaben mehr als einen Fall von assistiertem Suizid in den vergangenen 24 Monaten an.
- Tab. 2 Angaben zur Soziodemografie und Arbeitssituation in beiden Stichproben.

\begin{tabular}{|c|c|c|}
\hline & $\begin{array}{l}\text { Ärztinnen } \\
\text { und Ärzte }\end{array}$ & $\begin{array}{l}\text { Pflegerinnen } \\
\text { und Pfleger }\end{array}$ \\
\hline \multicolumn{3}{|l|}{ soziodemografische Angaben } \\
\hline Geschlecht, weiblich (\%) & $687(27,56)$ & $2319(86,59)$ \\
\hline \multicolumn{3}{|l|}{ Familienstand (\%) } \\
\hline ledig & $199(7,99)$ & $1324(49,97)$ \\
\hline verheiratet/Partnerschaft & $2095(84,14)$ & $1227(46,15)$ \\
\hline getrennt/geschieden/verwitwet & $196(7,87)$ & $108(4,06)$ \\
\hline Alter, Jahre, M (SD) & $52,59(8,39)$ & $29,76(11,22)$ \\
\hline Berufserfahrung, Jahre, M (SD) & $25,50(8,77)$ & $9,47(10,18)$ \\
\hline Belastungsfaktoren (24 Monate) & $\mathrm{M}(\mathrm{SD})$ & $\mathrm{M}(\mathrm{SD})$ \\
\hline berufliche Zufriedenheit & $3,73(0,74)$ & $2,96(0,70)$ \\
\hline Unterstützung durch Team & $4,00(0,64)$ & $3,58(0,64)$ \\
\hline Arbeitsbelastung & $3,06(0,71)$ & $3,60(0,62)$ \\
\hline
\end{tabular}

$M=$ Mittelwert; $S D=$ Standardabweichung. Alle statistischen Vergleiche zwischen beiden Berufsgruppen ( $X^{2}$ - und t-Tests) waren bei $p<0,001$ signifikant. Subjektiven Angaben zur Arbeitsbelastung wurden auf Skalen von 1 (unzufrieden/trifft überhaupt nicht zu) bis 5 (zufrieden/trifft voll und ganz zu) beantwortet, der Skalenmittelwert lag jeweils bei 3 .

\section{Befürwortung von aktiver Sterbehilfe}

Über die Hälfte der Pflegerinnen und Pfleger waren in den vergangenen 24 Monaten in mindestens einem konkreten Fall der Auffassung, aktive Sterbehilfe sei besser „z. B. um ihn/sie von seinem/ihrem Leid zu erlösen“, demgegenüber waren es nur etwa ein Viertel der Ärztinnen und Ärzte ( $\vee$ Tab. 3). Eine generelle Straffreiheit von aktiver Sterbehilfe wurde von den meisten Befragten abgelehnt, am stärksten durch Ärztinnen und Ärzte. Eine teilweise Zustimmung gaben lediglich die Pflegerinnen und Pfleger bei aktiver Sterbehilfe durch Ärztinnen und Ärzte an.

\section{Durchführung von aktiver Sterbehilfe}

Aktive Sterbehilfe wurde in den vergangenen 24 Monaten in der ärztlichen Berufsgruppe in 1,94\% der Fälle und in der pflegerischen Berufsgruppe in 1,24\% der Fälle praktiziert ( $\vee$ Tab.4).

In den vergangenen 24 Monaten hatten Ärzte und Ärztinnen in 84 Fällen und Pfleger und Pflegerinnen in 65 Fällen aktive Sterbehilfe praktiziert ( $\vee$ Tab.3). Während des gesamten Beschäftigungszeitraums hatten 278 Ärzte und Ärztinnen und 117 Pfleger und Pflegerinnen das Leben eines Patienten aktiv beendet ( $\triangleright$ Tab.3). Im Mittel gaben Befragte in beiden Gruppen 2 Fälle von aktiver Sterbehilfe in den vergangenen 24 Monaten an. Im letzten Fall von selbst durchgeführter aktiver Sterbehilfe wurde dies durch das Zurückhalten bzw. den Entzug einer Behandlung (77,49\%, 276 Fälle) mit dem Ziel der Lebensbeendigung oder durch Gabe eines Medikaments (22,51\%, 99 Fälle) bewirkt. In etwa der Hälfte der Fälle (48,49\%, 177 Fälle) sei der Patient nicht 
- Tab. 3 Angaben aus beiden Stichproben, inwieweit verschiedene Formen von sog. Sterbehilfe befürwortet, erlebt und selbst durchgeführt wurden.

\begin{tabular}{|c|c|c|c|}
\hline & $\begin{array}{l}\text { Ärztinnen } \\
\text { und Ärzte }\end{array}$ & $\begin{array}{l}\text { Pflegerinnen } \\
\text { und Pfleger }\end{array}$ & $\mathbf{p}$ \\
\hline $\begin{array}{l}\text { Befürwortung von } \\
\text { aktiver Sterbehilfe... }\end{array}$ & $M(S D)$ & $M(S D)$ & \\
\hline ...insgesamt & $1,71(0,66)$ & $2,55(0,95)$ & $<0,001^{*}$ \\
\hline $\begin{array}{l}\text {...durch Pflegerinnen/ } \\
\text { Pfleger }\end{array}$ & $1,22(0,52)$ & $2,05(1,06)$ & $<0,001^{*}$ \\
\hline $\begin{array}{l}\text {...durch Ärztinnen/ } \\
\text { Ärzte }\end{array}$ & $2,21(1,03)$ & $3,05(1,05)$ & $<0,001^{*}$ \\
\hline $\begin{array}{l}\text {...auch ohne explizite } \\
\text { Willenserklärung }\end{array}$ & $1,45(0,64)$ & $2,07(1,06)$ & $<0,001^{*}$ \\
\hline $\begin{array}{l}\text {...in mind. einem Fall, } \\
24 \text { Monate, } \mathrm{n}(\%)\end{array}$ & $607(24,23)$ & $1431(53,52)$ & $<0,001^{*}$ \\
\hline $\begin{array}{l}\text { Sterbehilfe (in den } \\
\text { letzten } 24 \text { Monaten) }\end{array}$ & $\begin{array}{l}\text { in mind. } \\
\text { einem Fall } \\
\mathrm{n}(\%)\end{array}$ & $\begin{array}{l}\text { in mind. } \\
\text { einem Fall } \\
\mathrm{n}(\%)\end{array}$ & $<0,001^{*}$ \\
\hline passive Sterbehilfe & $1083(56,02)$ & $870(35,01)$ & $<0,001^{*}$ \\
\hline indirekte Sterbehilfe & $671(27,38)$ & $753(30,33)$ & $0,02^{*}$ \\
\hline assistierter Suizid & $7(0,29)$ & $24(0,98)$ & $0,002^{*}$ \\
\hline $\begin{array}{l}\text { andere Methode } \\
\text { (Tod unbeabsichtigt) }\end{array}$ & $101(4,15)$ & $159(6,49)$ & $<0,001^{*}$ \\
\hline aktive Sterbehilfe & & & \\
\hline $\begin{array}{l}\text {...in den vergangenen } \\
24 \text { Monaten, andere }\end{array}$ & $224(8,93)$ & $272(10,14)$ & 0,12 \\
\hline $\begin{array}{l}\text {...in den vergangenen } \\
24 \text { Monaten, selbst }\end{array}$ & $84(3,35)$ & $65(2,42)$ & $0,05^{*}$ \\
\hline $\begin{array}{l}\ldots \text { in gesamter } \\
\text { Tätigkeit, selbst }\end{array}$ & $278(11,04)$ & $117(4,17)$ & $<0,001^{*}$ \\
\hline $\begin{array}{l}\text { davon: Methode im } \\
\text { letzten Fall; durch... }\end{array}$ & & & $0,006^{*}$ \\
\hline $\begin{array}{l}\text {...Zurückhalten/Ent- } \\
\text { zug v. Behandlung }\end{array}$ & $210(77,49)$ & $66(62,85)$ & \\
\hline ...Gabe v. Medikament & $61(22,51)$ & $38(36,19)$ & \\
\hline ....andere Methode & $0(0,00)$ & $1(0,95)$ & \\
\hline \multicolumn{4}{|c|}{$\begin{array}{l}\text { M = Mittelwert; SD = Standardabweichung. } \\
\text { " gibt signifikante Unterschiede zwischen den Berufsgruppen an, der } \\
\text { p-Wert (für } X^{2} \text { - und t-Tests) ist jeweils angegeben. Die Einstellung } \\
\text { gegenüber einer Straffreiheit von aktiver Sterbehilfe wurde auf einer } \\
\text { Skala von } 1 \text { (stimme überhaupt nicht zu) bis } 5 \text { (stimme voll und ganz } \\
\text { zu) beantwortet, der Skalenmittelwert lag bei 3. Abweichungen von } \\
\text { Summenwerten durch fehlende Angaben. Bei Prozentwerten sind } \\
\text { fehlende Werte unberücksichtigt. }\end{array}$} \\
\hline
\end{tabular}

mehr in der Lage dazu gewesen, sich zu äußern. Bei diesen habe für die meisten eine explizite Willensbekundung von Patientenseite (74,58\%, 132 Fälle) oder (zusätzlich) durch Angehörige (56,50\%, 100 Fälle) vorgelegen. Bei etwa einem Drittel der äußerungsfähigen Patientinnen und Patienten (17,39\%, 32 Fälle) habe weder eine Willensäußerung durch sie selbst noch durch ihre An-
- Tab. 4 Fälle selbst durchgeführter Sterbehilfe in den vergangenen 24 Monaten.

\begin{tabular}{|l|l|l|}
\hline & $\begin{array}{l}\text { Ärztinnen } \\
\text { und Ärzte }\end{array}$ & $\begin{array}{l}\text { Pflegerinnen } \\
\text { und Pfleger }\end{array}$ \\
\hline & $\mathbf{n}(\mathbf{\%})$ & $\mathbf{n}(\%)$ \\
\hline $\begin{array}{l}\text { gesamte Fälle (in den letzten } \\
\text { 24 Monaten) }\end{array}$ & $23648(100,00)$ & $17771(100,00)$ \\
\hline $\begin{array}{l}\text { passive Sterbehilfe } \\
\text { indirekte Sterbehilfe }\end{array}$ & $14799(62,58)$ & $8528(47,99)$ \\
\hline andere Methode & $7554(31,94)$ & $8045(45,27)$ \\
\hline \begin{tabular}{l} 
assistierter Suizid \\
\hline aktive Sterbehilfe
\end{tabular} & $778(3,29)$ & $902(5,08)$ \\
\hline
\end{tabular}

Fallzahlen basieren auf Angaben der Befragten zur eigenen Durchführung von Sterbehilfe in den vergangenen 24 Monaten.

gehörigen vorgelegen. Dies war ebenso bei 45 Patientinnen und Patienten der Fall, die nicht äußerungsfähig gewesen seien $(25,42 \%)$.

\section{Erklärung des Auftretens von aktiver Sterbehilfe in den vergangenen 24 Monaten}

Unter statistischer Berücksichtigung der jeweils anderen Variablen im Modell zeigten sich die Arbeitsbedingungen, insbesondere der Stationstyp (abgeleitet vom Fachgebiet) $(O R=5,88$; $95 \%-K I=2,81-1228)$, und die ärztliche Berufsgruppe ( $O R=2,67$; $95 \%-K I=1,39-5,11)$ als die stärksten Einflussfaktoren auf die Durchführung aktiver Sterbehilfe in den vergangenen 24 Monaten ( $\triangleright$ Tab. 5). Varianz in ausgeübter aktiver Sterbehilfe konnte zudem u. a. durch einen häufigeren Kontakt zu sterbenden Patientinnen und Patienten $(O R=1,24 ; 95 \%-K I=1,24-1,78)$ erklärt werden, nicht jedoch durch die danach geäußerten Wünsche von Patienten-/Angehörigenseite ( $O R=1,00 ; 95 \%-K I=0,99-1,01)$. Die Straffreiheit von aktiver Sterbehilfe stärker zu befürworten ging im gleichen Zeitraum auch mit deren vermehrter Anwendung einher $(O R=2,09 ; 95 \%-K I=1,68-2,60)$. Gleiches galt, wenn man diese bei anderen mitbekommen hatte $(O R=1,21$; $95 \%-K I=1,17-1,26)$. Die Durchführung passiver Sterbehilfe zeigte bei Berücksichtigung der übrigen Modellvariablen einen nur geringen, jedoch tendenziell verringernden Einfluss auf die Durchführung aktiver Sterbehilfe (OR=0,97; $95 \%-K I=0,95-1,00)$. Keiner der subjektiven Belastungsfaktoren in den vergangenen 24 Monaten zeigte einen Einfluss auf die Durchführung aktiver Sterbehilfe im selben Zeitraum.

\section{Diskussion}

Die vorliegende Studie konnte das Vorkommen von allen Formen der Sterbehilfe durch die ärztliche und pflegerische Berufsgruppe in deutschen Krankenhäusern zeigen. Innerhalb von 24 Monaten führten nach eigenen Angaben etwa ein Drittel der Pflegerinnen 
- Tab. 5 Multivariable logistische Regression der aktiven Sterbehil$\mathrm{fe}$ in den vergangenen 24 Monaten.

\begin{tabular}{|c|c|c|}
\hline Prädiktor & $\begin{array}{l}\text { Odds } \\
\text { Ratio }\end{array}$ & $95 \%-K I$ \\
\hline \multicolumn{3}{|l|}{ Person/Arbeitsbedingungen } \\
\hline Geschlecht (männlich) & $1,68^{*}$ & $1,01-2,81$ \\
\hline Berufsgruppe (ärztlich) & $2,67^{*}$ & $1,39-5,11$ \\
\hline Berufserfahrung & 1,00 & $0,98-1,02$ \\
\hline Stationstyp (Fachgebiet) & $5,88^{* *}$ & $2,81-12,28$ \\
\hline Kontakt zu sterbenden Patienten & $1,49^{* *}$ & $1,24-1,78$ \\
\hline $\begin{array}{l}\text { geäußerter Wunsch nach aktiver } \\
\text { Sterbehilfe }\end{array}$ & 1,00 & 0,99-1,01 \\
\hline \multicolumn{3}{|l|}{ palliative Maßnahmen } \\
\hline passive Sterbehilfe & $0,97^{*}$ & $0,95-1,00$ \\
\hline indirekte Sterbehilfe & 0,99 & $0,97-1,02$ \\
\hline \multicolumn{3}{|l|}{ Belastungsfaktoren } \\
\hline berufliche Zufriedenheit & 1,04 & $0,72-1,52$ \\
\hline Unterstützung im Team & 1,23 & $0,86-1,75$ \\
\hline Arbeitsbelastung & 1,15 & $0,80-1,66$ \\
\hline \multicolumn{3}{|l|}{ Einstellung/Einflüsse anderer } \\
\hline $\begin{array}{l}\text { aktive Sterbehilfe durch Kollegen } \\
\text { beobachtet }\end{array}$ & $1,21^{* *}$ & $1,17-1,26$ \\
\hline Befürwortung von aktiver Sterbehilfe & $2,09^{* *}$ & $1,68-2,60$ \\
\hline \multicolumn{3}{|c|}{$\begin{array}{l}\text { Signifikantes Gesamtmodell } X^{2}(13)=344,76 ; p<0,001 \text {; Nagelkerkes } \\
R^{2}=0,30 \text {; korrekte Klassifikation von } 97,33 \% \text { der Befragten }(14,60 \% \\
\text { korrekte Zuordnung zur aktiven Sterbehilfe). } \\
{ }^{*}<0,05 \text {. } \\
\quad<0,001 \text {. }\end{array}$} \\
\hline
\end{tabular}

und Pfleger und die Hälfte der Ärztinnen und Ärzte indirekte bzw. passive Sterbehilfe durch (38926 berichtete Fälle). Der Anteil von durchgeführter aktiver Sterbehilfe war im gleichen Zeitraum deutlich geringer (680 berichtete Fälle).

Die aktuelle Studie ergänzt die wenigen Befunde zur Durchführung von verschiedenen Formen von Sterbehilfe in Deutschland. Sie bestätigt die Ergebnisse einer Studie aus dem Jahr 2017. Damals gaben - je nach Berufsgruppe - zwischen 1,42 und 3,39\% der Befragten an, schon einmal selbst aktive lebensbeendende Handlungen durchgeführt zu haben [7]. Die berichteten Angaben liegen unterhalb der Vergleichswerte von Maitra et al. [4], bei denen $13 \%$ der niedergelassenen Hausärztinnen und -ärzte angaben, bereits aktive Sterbehilfe durchgeführt zu haben, jedoch über denen von Schildmann et al. [17] innerhalb von 12 Monaten. Die Ausübung von assistiertem Suizid wird bei Jansky et al. [18] mit einem höheren Anteil von 3,2-3,6\% der (überwiegend palliativmedizinisch tätigen) Ärztinnen und Ärzten sowie anderen Berufsgruppen angegeben. Abweichende Beobachtungszeiträume und Fragebogenmethodik berechtigen jedoch zu keinem direkten Vergleich.

Die gleichzeitige Befragung von Pflegerinnen und Pflegern konnte deren Beteiligung an allen Formen von Sterbehilfe bele- gen, wie dies auch aus Studien aus anderen Ländern bekannt ist [19]. Ein direkter Vergleich mit der ärztlichen Berufsgruppe wird jedoch durch Unterschiede zwischen den Stichproben erschwert. Obwohl es sich um eine relativ große Stichprobe handelt, lassen sich keine generalisierbaren Aussagen zur Praxis der Sterbehilfe in deutschen Kliniken treffen. Dazu sind repräsentative Stichproben erforderlich [14], die besonders beachten, dass ein wesentlicher Unterschied zwischen aktiver Sterbehilfe auf der einen Seite und indirekter bzw. passiver Sterbehilfe auf der anderen Seite in der Handlungsmotivation besteht. Bei der aktiven Sterbehilfe ist die Lebensbeendigung primär beabsichtigte Folge einer Handlung, bei anderen Formen von Sterbehilfe ist dagegen eine eventuelle Lebensverkürzung primär nicht beabsichtigt. Daher ließ diese Studie bei der Abfrage von angewendeten Maßnahmen nachdem die Durchführung von aktiver Sterbehilfe bejaht wurde - die Angabe zu, eine absichtliche Lebensbeendigung auch durch das Zurückhalten bzw. den Entzug von Behandlung zu bewirken ( $\vee$ Tab.3). Hier könnte durch die nicht definitionskonforme Zuordnung eine zuverlässige Antwortentscheidung erschwert worden sein. Eine Limitation der Studie stellen sozial erwünschte Antwortverzerrungen und die Nachteile von Online-Befragungen dar [20]. Angesichts eines möglichen Antwortbias durch die Brisanz einiger Angaben sollte die Interpretation mit Vorsicht erfolgen. Trotz Eingrenzung auf einen 24-monatigen Zeitraum lässt die querschnittliche Natur der Daten keine kausalen Schlüsse seitens der Einflussfaktoren auf die Durchführung von aktiver Sterbehilfe zu.

Zukünftige Forschung sollte weiterhin die Gruppe der Pflegekräfte in Befragungen zum Thema Sterbehilfe einbeziehen und verschiedene Formen von Sterbehilfe gemeinsam erheben. Die Konstruktion von sensitiven, begrifflich neutralen Fragebögen, Wahrung der Anonymität und Zusammenarbeit mit den Ärzteund Pflegekammern sind Voraussetzung für verlässliche, repräsentative Daten, von denen auch die gesellschaftliche Debatte in Deutschland profitieren könnte.

Forschung zur Lage in den Niederlanden nach Inkrafttreten der Gesetzesreform 2002 zeigte einen nur moderaten Anstieg von aktiver Sterbehilfe [21], worauf von Befürwortern einer entsprechenden Gesetzesänderung in Deutschland hingewiesen wird. Gleichzeitig zeigen die Befunde der vorliegenden Studie einen nur geringen Zusammenhang zwischen der Anwendung verschiedener Formen von Sterbehilfe und ihren jeweiligen rechtlichen Konsequenzen. So wurde der zumindest in Einzelfällen straffreie assistierte Suizid deutlich seltener angegeben als die verbotene aktive Sterbehilfe. Dies wirft die Frage auf, welchen Einfluss vorhandene oder zukünftige Gesetze auf die individuellen Entscheidungen von ärztlichen und pflegerischen Mitarbeitern in Krankenhäusern nehmen können. Tatsächlichen Einfluss hierauf nahmen die Anwendung palliativmedizinischer Kenntnisse, das beobachtete Verhalten von Kolleginnen und Kollegen sowie die (überwiegend ablehnende) eigene Haltung gegenüber aktiver Sterbehilfe. Entsprechende Förderung palliativmedizinischer Weiterbildungen für alle medizinischen Berufsgruppen mit häufigem Kontakt zu Sterbenden und ein offener Austausch unter ihnen könnten daher sinnvolle Möglichkeiten darstellen, um im Spannungsfeld von Selbstbestimmung und Fürsorge am Lebensende [22] eine humane Sterbebegleitung zu verwirklichen. 


\section{KERNAUSSAGEN}

- Passive bzw. indirekte Sterbehilfe wurde innerhalb von 24 Monaten von ca. der Hälfte der befragten Ärztinnen und Ärzte und einem Drittel der Pflegerinnen und Pfleger in deutschen Krankenhäusern berichtet.

- Aktive Sterbehilfe und assistierter Suizid wurden von einem geringen Teil beider Berufsgruppen berichtet.

- Variation in der Anwendung von aktiver Sterbehilfe wurde durch arbeitsbezogene Faktoren, die Befürwortung aktiver Sterbehilfe und durch das beobachtete Verhalten anderer im selben Zeitraum beeinflusst.

- Durchgeführte passive Sterbehilfe wirkte sich verringernd auf praktizierte aktive Sterbehilfe aus; es gab in der vorliegenden Studie keinen Zusammenhang zu subjektiver Arbeitsbelastung.

- Repräsentative Stichproben sind notwendig, um die vorliegenden Ergebnisse zu replizieren.

\section{Interessenkonflikt}

Die Autorinnen/Autoren geben an, dass kein Interessenkonflikt besteht.

\section{Danksagung}

Der Autor dankt allen Ärztinnen und Ärzten sowie Pflegerinnen und Pflegern, die an der Befragung teilgenommen haben, und Dr. Torben Schubert und Michaela Assheuer für ihre Forschungsassistenz.

\section{Literatur}

[1] Bundesverfassungsgericht, Urteil vom 26.02.2020, AZ: 2 BvR 2347/15, 2 BvR 651/16, 2 BvR 1261/16, 2 BvR 1593/16, 2 BvR 2354/16, 2 BVR 2527/16.

[2] Jacob N. Aktive Sterbehilfe im Rechtsvergleich und unter der Europäischen Menschenrechtskonvention. s.l.: Tectum Wissenschaftsverlag. 2014

[3] Monforte-Royo C, Villavicencio-Chávez C, Tomás-Sábado J et al. The wish to hasten death. A review of clinical studies. Psycho-Oncology 2011; 20: 795-804. doi:10.1002/pon.1839

[4] Maitra RT, Harfst A, Bjerre LM et al. Do German General Practitioners Support Euthanasia? European Journal of General Practice 2010; 11: 94100. doi:10.3109/13814780509178247

[5] Schildmann J, Hoetzel J, Mueller-Busch C et al. End-of-life practices in palliative care. A cross sectional survey of physician members of the German Society for Palliative Medicine. Palliat Med 2010; 24: 820-827. doi:10.1177/0269216310381663

[6] Hagelin J, Nilstun T, Hau J et al. Surveys on attitudes towards legalisation of euthanasia. Importance of question phrasing. J Med Ethics 2004; 30: 521-523. doi:10.1136/jme.2002.002543
[7] Beine KH, Schubert T. Das Dunkelfeld intentional lebensbeendender Handlungen durch Ärzte und Pflegekräfte. Dtsch Med Wochenschr 2017; 142: e83-e88. doi:10.1055/s-0043-109889

[8] Smets T, Cohen J, Bilsen J et al. The labelling and reporting of euthanasia by Belgian physicians: a study of hypothetical cases. Eur J Public Health 2012; 22: 19-26. doi:10.1093/eurpub/ckq180

[9] Strack F. "Order Effects" in Survey Research: Activation and Information Functions of Preceding Questions. In: Schwarz N, Sudman S, eds.; Context Effects in Social and Psychological Research. New York, NY: Springer New York; 1992: 23-34. doi:10.1007/978-1-4612-2848-6_3

[10] Seale C. National survey of end-of-life decisions made by UK medical practitioners. Palliat Med 2016; 20: 3-10. doi:10.1191/ $0269216306 \mathrm{pm} 10940 \mathrm{oa}$

[11] Löfmark R, Nilstun T, Cartwright C et al. Physicians' experiences with end-of-life decision-making. Survey in 6 European countries and Australia. BMC Med 2008; 6: 4. doi:10.1186/1741-7015-6-4

[12] Meier DE, Emmons CA, Wallenstein S et al. A national survey of physician-assisted suicide and euthanasia in the United States. N Engl J Med 1998; 338: 1193-1201. doi:10.1056/NEJM199804233381706

[13] Mortier F, Bilsen J, Vander Stichele RH et al. Attitudes, sociodemographic characteristics, and actual end-of-life decisions of physicians in Flanders, Belgium. Medical decision making 2003; 23: 502-510. doi:10.1177| $0272989 \times 03260137$

[14] Farber NJ, Urban SY, Collier VU et al. Frequency and perceived competence in providing palliative care to terminally ill patients. A survey of primary care physicians. J Pain Symptom Manage 2004; 28: 364-372. doi:10.1016/j.jpainsymman.2004.01.013

[15] Aiken LH, Clarke SP, Sloane DM et al. Hospital nurse staffing and patient mortality, nurse burnout, and job dissatisfaction. JAMA 2002; 288: 1987-1993. doi:10.1001/jama.288.16.1987

[16] Böttger-Kessler G, Beine KH. Aktive Sterbehilfe bei Menschen im Wachkoma? Ergebnisse einer Einstellungsuntersuchung bei Ärzten und Pflegenden. Nervenarzt 2007; 78: 802-808. doi:10.1007/s00115-0062241-5

[17] Schildmann J, Dahmen B, Vollmann J. Ärztliche Handlungspraxis am Lebensende. Dtsch Med Wochenschr 2015; 140: e1-e6. doi:10.1055/ s-0034-1387410

[18] Jansky M, Jaspers B, Radbruch L et al. Einstellungen zu und Erfahrungen mit ärztlich assistiertem Suizid. Bundesgesundheitsbl 2017; 60: 89-98. doi:10.1007/s00103-016-2476-7

[19] Bilsen JJR, Vander Stichele RH, Mortier F et al. Involvement of nurses in physician-assisted dying. J Adv Nurs 2004; 47: 583-591. doi:10.1111/ j.1365-2648.2004.02982.x

[20] Lefever S, Dal M, Matthíasdóttir Á. Online data collection in academic research. Advantages and limitations. Br J Educ Technol 2007; 38: 574582. doi:10.1111/j.1467-8535.2006.00638.x

[21] Onwuteaka-Philipsen BD, Brinkman-Stoppelenburg A, Penning C et al. Trends in end-of-life practices before and after the enactment of the euthanasia law in the Netherlands from 1990 to 2010. A repeated crosssectional survey. The Lancet 2012; 380: 908-915. doi:10.1016/ S0140-6736(12)61034-4

[22] Nationaler Ethikrat. Selbstbestimmung und Fürsorge am Lebensende 2006. Im Internet: https://www.ethikrat.org/fileadmin/Publikationen/ Stellungnahmen/Archiv/Stellungnahme_Selbstbestimmung_und_ Fuersorge_am_Lebensende.pdf 